Document downloaded from:

http://hdl.handle.net/10251/57067

This paper must be cited as:

Montoliu, C.; Ferrando Jódar, N.; Gosalvez Ayuso, MA.; Cerdá Boluda, J.; Colom Palero, RJ. (2013). Level set implementation for the simulation of anisotropic etching: application to complex MEMS micromachining. Journal of Micromechanics and Microengineering. 23(7). doi:10.1088/0960-1317/23/7/075017.

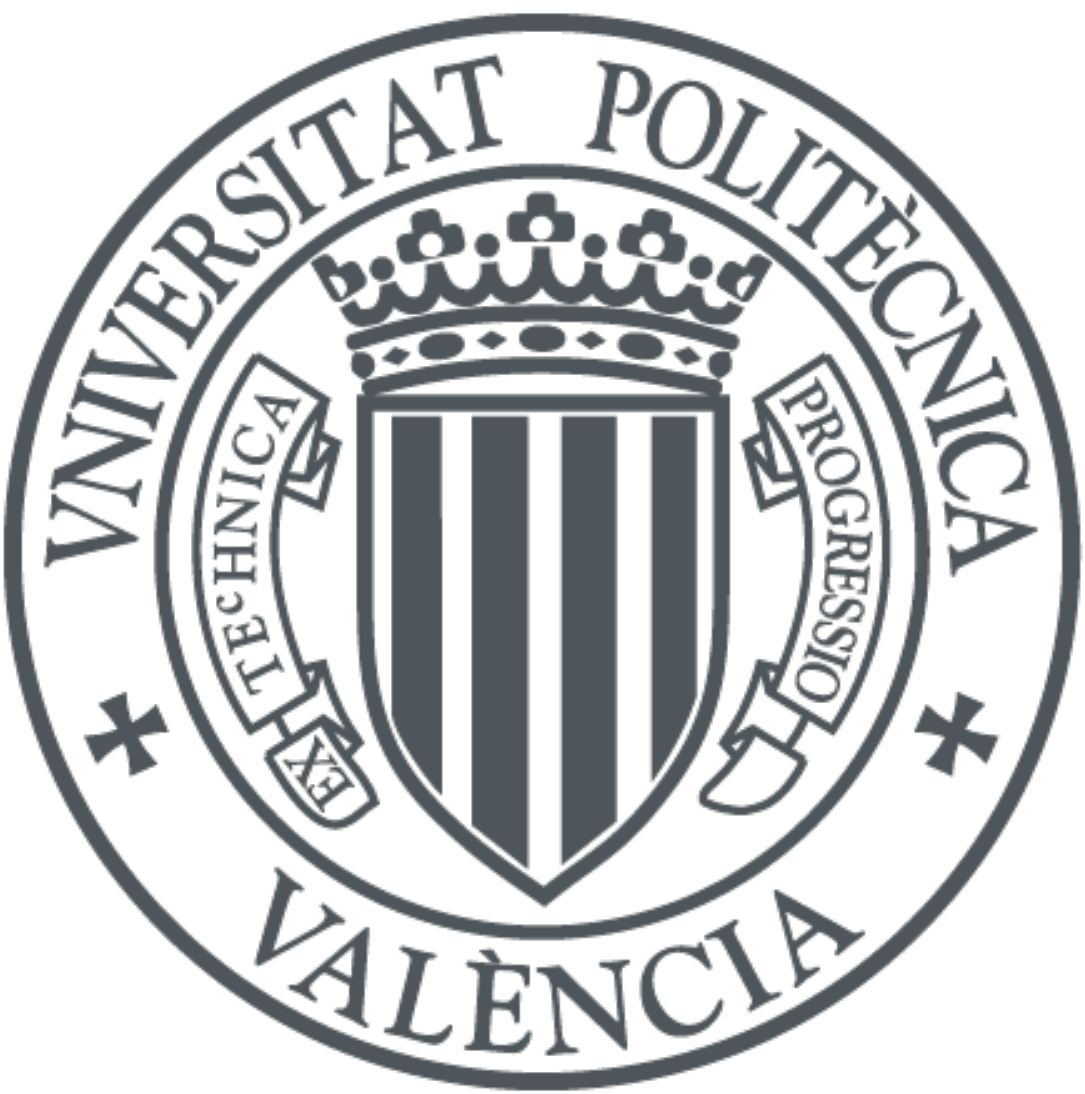

The final publication is available at

http://dx.doi.org/10.1088/0960-1317/23/7/075017

Copyright IOP Publishing: Hybrid Open Access

Additional Information 


\title{
Level Set implementation for the simulation of anisotropic etching: Application to complex MEMS micromachining
}

\author{
C. Montoliu ${ }^{1}$, N. Ferrando ${ }^{2}$, M. A. Gosálvez ${ }^{3}$, J. Cerdá ${ }^{1}$ and R. \\ J. Colom ${ }^{1}$ \\ ${ }^{1}$ Instituto de Intrumentación para Imagen Molecular, Universitat Politécnica de \\ Valéncia, Camino de Vera s/n, 46022 Valencia, Spain \\ ${ }^{2}$ Centro de Física de Materiales, centro mixto CSIC-UPV/EHU and Donostia \\ International Physics Center (DIPC) 20018 Donostia-San Sebastian, Spain \\ ${ }^{3}$ Department of Materials Physics, University of the Basque Country (UPV-EHU), \\ Donostia International Physics Center (DIPC), 20018 Donostia-San Sebastian, Spain \\ E-mail: carmonal@upv.es
}

\begin{abstract}
.
The use of atomistic methods, such as the Continuous Cellular Automaton (CCA), is currently regarded as an accurate and efficient approach for the simulation of anisotropic etching in the development of Micro-Electro-Mechanical-Systems (MEMS). However, whenever the targeted etching condition is modified (e.g. by changing the substrate material, etchant type, concentration and/or temperature) this approach requires performing a time-consuming recalibration of the full set of internal atomistic rates defined within the method. Based on the Level Set (LS) approach as an alternative and using the experimental data directly as input, we present a fully operational simulator that exhibits similar accuracy than the latest CCA models. The proposed simulator is tested by describing a wide range of silicon and quartz MEMS structures obtained in different etchants through complex processes, including doubleside etching as well as different mask patterns during different etching steps and/or simultaneous masking materials on different regions of the substrate. The results demonstrate that the LS method is able to simulate anisotropic etching for complex MEMS processes with similar computational times and accuracy as the atomistic models.
\end{abstract}

PACS numbers: 89.20.Ff, 85.85.+j, 81.65.Cf

Keywords: Anisotropic wet etching, Level Set, Sparse Field Method, Microengineering, Cellular Automata, Micro-electro-mechanical systems, MEMS, Parallel computing Submitted to: J. Micromech. Microeng. 


\section{Introduction}

Anisotropic wet chemical etching of crystalline silicon is one of the most popular methods for the production of Micro-Electro-Mechanical Systems (MEMS). The key aspects of the process are its low cost, the ability to generate smooth surfaces, the possibility to release suspended structures and the simplicity to perform batch fabrication. Nevertheless, the particular shapes of the resulting three-dimensional structures depend on multiple factors, such as the crystallographic orientation of the surface $[1,2,3,4,5,6]$, the composition of the applied etchant - e.g. potassium hydroxide $(\mathrm{KOH})[7,8]$, tetramethylammonium hydroxide (TMAH) $[9,10]$ or various other solutions [11]- the etchant concentration $[4,6]$, the temperature $[2,4,6]$ or the use of additives, such as Triton X$100[12,13]$ or isopropyl alcohol $[4,8,14]$. Correspondingly, significant efforts have been devoted by the MEMS community throughout the years to characterize and simulate the different features of the process as accurately and efficiently as possible.

Based on geometrical models, the first simulators described wet etching as a set of moving facets $[15,16]$. This posed several challenges, especially the efficient implementation of the intersection of two or more planes in 3D and, correspondingly, the description of wafer perforation. As an alternative, the use of atomistic approaches, such as Cellular Automata (CA) [17, 18, 19, 20, 21, 22, 23, 24] and Kinetic Monte Carlo (KMC) [25, 26, 27, 28], has become gradually more successful. The substrate is described as a crystallographic collection of atoms and the etch front is treated as the corresponding subset of surface atoms, which are removed according to simple, neighborhood-dependent removal rates. This results in the emergence of the neighboring atoms to the surface, automatically enabling the description of the splitting and coalescing of disjoint regions of the etch front without any further computational/programming effort. Although KMC has been used traditionally to understand the origin of the surface roughness on different substrate orientations [26, 27], there exist successful examples of its use for microstructure prediction $[20,28]$. In turn, $\mathrm{CA}$ is best suited for modeling the formation of 3D microstructures, but it has also been used to describe a wide range of surface morphologies [29].

Although the first CA models were based on stochastic approaches [17], they eventually gave rise to various deterministic procedures [18, 19, 20]. Based on the step flow nature of wet etching $[5,22]$, the Continuous CA (CCA) method has demonstrated foremost flexibility and accuracy, simulating reliably wet etching for a wide range of MEMS structures and conditions [21, 30]. Moreover, algorithmic accelerations for sequential and parallel computational environments have been developed [23, 24] including commercial simulators [31]- enabling the execution of the CCA calculations on Graphics Processing Units (GPUs) and drastically reducing the computation times to just a few seconds per simulation. An additional virtue over the geometrical methods is the possibility of calibrating the atomistic model parameters by using a reduced dataset of experimental etch rates [32]. Originally requiring careful supervision by the user, the calibration process has been recently automated by using an evolutionary algorithm 
for both KMC [28] and CCA [33, 34]. Despite the good results, new calibrations are needed each time the experimental conditions are changed, such as the etchant type, concentration and/or temperature, requiring several hours or even days for calibration completion. In addition, a complete reclassification of the neighborhood-dependent rates is required if the crystallographic structure of the substrate is changed (e.g. silicon vs quartz) [34]. In this context, the search of an alternative method, which is simultaneously capable of using the experimental data without prior calibration while remaining computationally efficient, has the potential to influence significantly the future of MEMS design.

In this study we consider the use of the Level Set (LS) method, originally introduced for capturing moving fronts [35] and later developed for related problems, such as chemical etching [36, 37, 38, 39], Deep Reactive Ion Etching (DRIE) [40], abrasive jet micromachining [41] and plasma etching [42]. While LS has been previously reported as a successful method for simulating the anisotropy of $\mathrm{KOH}$ etching [43, 44, 45, 46], a more exhaustive evaluation is required. We present an implementation that is capable of simulating anisotropic etching on double-sided wafers of any material as well as advanced processes, such as the sequential use of different mask patterns during successive etching steps and/or the simultaneous use of different masking materials on different regions of the substrate. Benefitting from the direct use of the etch rates obtained from the experiments, the proposed LS implementation is extensively examined by simulating the formation of a wide variety of complex, realistic MEMS structures based on silicon and quartz substrates in different etchants, comparing the predicted shapes to those from experiments and state-of-the-art CCA simulations. Also, a brief comparison of the computational times of both methods is given.

\section{Level set method applied to anisotropic wet etching}

The LS method was introduced by Sethian et al. as a computational approach for easily modeling moving fronts [35]. Given a front $x(t)$ at time $t$ in an $n$-dimensional space $S$, the main idea is to consider the signed distance $(\phi)$ from the front to any point in $S$, so that by construction the front will always remain as the zero hypersurface (or zero level set) of $\phi$ at any time: $\phi(x(t))=0$. The advantage of embedding the front $x(t)$ inside a $(n+1)$-dimensional function $\phi$ is that it enables propagating directly $\phi$ instead of $x(t)$ so that the splitting and coalescing of disjoint regions of $x(t)$ can be described without any additional programming effort. Writing $\frac{d \phi(x(t))}{d t}=0$ and using the chain rule $\left(\frac{d}{d t}=\frac{\partial}{\partial t}+\sum_{i=1}^{n} \frac{\partial}{\partial x_{i}} \frac{\partial x_{i}}{\partial t}\right)$ gives rise to the fundamental Level Set Equation [35]:

$$
\phi_{t}+R(x(t), t)|\nabla \phi|=0,
$$

where $R(x(t), t)$ represents the propagation velocity in the normal direction. In general, $R$ may be a function of time and/or the actual geometry of the front, as in curvaturedependent problems. For anisotropic etching, $R$ is constant in time but orientationdependent, i.e. the rate of propagation of the front depends on the actual direction of 
the local normal to the front. In fact, the etch rates are described experimentally as a 180x45 matrix, $R(\theta, \Phi)$, in terms of the azimuth $(\Phi)$ and latitude $(\theta)$ of 8100 normal vectors in spherical coordinates with a resolution of 2 degrees [6].

Defining $H=R|\nabla \phi|$, we discretize Eq. 1 by using the following modified LaxFriedrichs scheme [47]:

$$
\begin{aligned}
& \phi_{i, j, k}^{n+1}=\phi_{i, j, k}^{n}-\Delta t\left[H\left(\beta_{x}^{+}, \beta_{y}^{+}, \beta_{z}^{+}\right)-\alpha_{F}\left(\alpha_{x} \beta_{x}^{-}+\alpha_{y} \beta_{y}^{-}+\alpha_{z} \beta_{z}^{-}\right)\right], \\
& \beta_{l}^{ \pm}=\frac{1}{2}\left(\frac{\phi_{(i, j, k)+1}^{n}-\phi_{(i, j, k)}^{n}}{\Delta l} \pm \frac{\phi_{(i, j, k)}^{n}-\phi_{(i, j, k)-1}^{n}}{\Delta l}\right) ; \\
& \alpha_{l}=\max \left|\frac{R\left(N_{l}+\Delta N_{l}, N_{p}, N_{q}\right)-R\left(N_{l}-\Delta N_{l}, N_{p}, N_{q}\right)}{2 \cdot \Delta N_{l}} \frac{\phi_{p}^{2}+\phi_{q}^{2}}{|\nabla \phi|^{2}}+R(\theta, \Phi) \cdot N_{l}\right|
\end{aligned}
$$

where $l=x, y, z$ and the operation $(i, j, k) \pm 1$ only affects the index $i, j$ or $k$ corresponding to the selected $l$. For simplicity, we take the spatial resolution of the grid to be equal in all dimensions $(\Delta l=\Delta x=\Delta y=\Delta z)$. The coefficients $\alpha_{l}$ are the Lax-Friedrichs artificial viscosity factors $\left(\alpha_{l}=\max \left|\frac{\partial H}{\partial \phi_{l}}\right|\right)$ and $\alpha_{F}$ is an overall viscosity introduced to guarantee the stability of the etch front. Larger $\alpha_{F}$ results in smoother fronts while smaller $\alpha_{F}$ generates sharp features/discontinuities. Depending on the anisotropy of the simulated etchant, different values of $\alpha_{F}$ are needed. We have found that $\alpha_{F}=0.48$ is adequate to simulate $\mathrm{KOH}$-based etchants, while $\alpha_{F}=0.45$ and $\alpha_{F}=0.5$ are more suitable to simulate TMAH-based and saturated $\mathrm{NH}_{4} \mathrm{HF}_{2^{-}}$ based etchants respectively. The procedure to obtain these parameters is trial and error, selecting those values which produce the most realistic results. Too large values result in soft, featureless fronts while too small values result in discontinuities and/or unnatural, sharp features. $N_{l}$ is the $l$-component of the unit normal vector with $l, p$ and $q$ any of $x$, $y$ and $z$, such that $p \neq l, q \neq l$ and $p \neq q$. Finally, $\frac{R\left(N_{l}+\Delta N_{l}, N_{p}, N_{q}\right)-R\left(N_{l}-\Delta N_{l}, N_{p}, N_{q}\right)}{2 \cdot \Delta N_{l}}$ is an approximation for $\frac{\partial R(\theta, \Phi)}{\partial N_{l}}$, determined by discretizing each component with a resolution of $\Delta N_{l}=0.01$.

Fig. 1 shows an example of the signed distance function $\phi$ for a simple twodimensional grid. The negative (positive) values of $\phi$ represent the substrate (etchant) regions while the black line designates the zero-level of $\phi$, which corresponds to the substrate-etchant interface. The local normal vector $\vec{N}_{i}$ is calculated at each grid point and directly associated an etch rate $R\left(\vec{N}_{i}\right)$ available from the experiments [6, 10]. In contrast to this straightforward use of the experimental data in the LS method, the CCA model requires transforming the experimental etch rates into internal atomistic rates, thus requiring a complete re-calibration of the actual atomistic rates or even a re-classification of the surface atom types when the experimental conditions are changed $[33,34]$.

Since the LS method has a large computational cost, $O\left(N^{3}\right)$, where $N$ is the number of grid points per dimension, the Sparse Field Method (SFM) was introduced to reduce the cost down to $O\left(N^{2}\right)$ by updating only the strictly necessary front points and nearest 


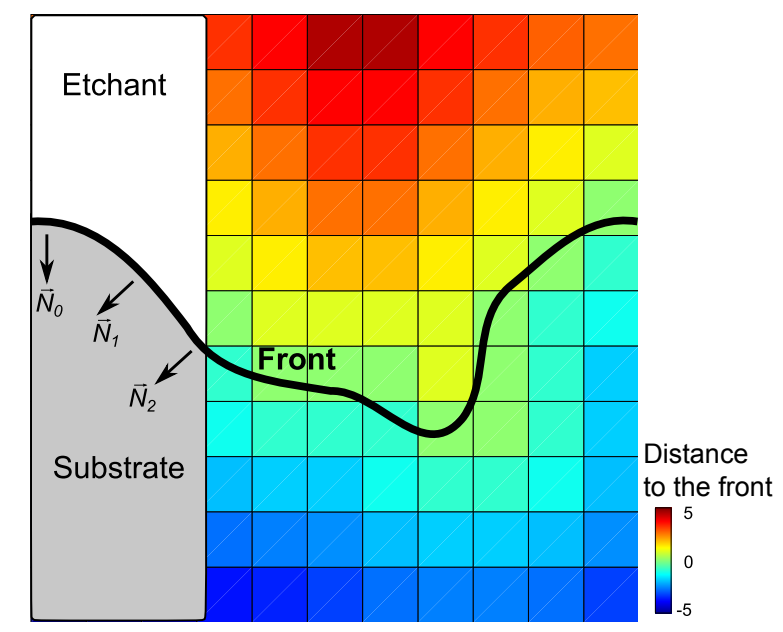

Figure 1. Two-dimensional example of the signed distance $\phi$ to the front (shown in black). The voxel colors represent the value of $\phi$. A few normals to the front are shown.

neighbors at each time step [48]. The particular SFM implementation used in this study is described in Fig. 2. The idea is to label all the points in $S$ according to five states ( 0 for points located at the front, +1 for nearest neighbors of the front located under the front, -1 for nearest neighbors located on the front, +2 for points located further away below the front and -2 for points further away above the front) and to create three processing lists $\left(L_{-1}, L_{0}\right.$ and $\left.L_{+1}\right)$ and their temporary counterparts $\left(S_{-1}, S_{0}\right.$ and $\left.S_{+1}\right)$. Given this structure, we loop through the points $(i, j, k)$ in $L_{0}$, updating $\phi$ by applying Eqs. 2-4 and placing into $S_{ \pm 1}$ those $(i, j, k)$ points whose distance to the front becomes larger than half the grid spacing. We then loop through the points $(i, j, k)$ in $L_{ \pm 1}$, updating $\phi_{i, j, k}^{n+1}=\phi_{i_{n}, j_{n}, k_{n}}^{n+1} \pm \Delta l$, where $\left(i_{n}, j_{n}, k_{n}\right)$ is the neighbor from $L_{0}$ that has the smallest $(+) /$ largest $(-) \phi_{i_{n}, j_{n}, k_{n}}^{n+1}$ value, while placing into $S_{0}$ those $(i, j, k)$ points whose distance to the front becomes smaller than half the grid spacing as well as removing those points $(i, j, k)$ whose distance value satisfies $\left|\phi_{i, j, k}^{n+1}\right|>1.5 \Delta l$. We finally update the lists $L_{-1}, L_{0}$ and $L_{+1}$ by (I) inserting the corresponding points from the temporary lists $S_{-1}, S_{0}$ and $S_{+1}$ (which become empty) and (II) performing a final loop over the points $(i, j, k)$ in $L_{0}$ in order to search for nearest neighbors $\left(i_{n}, j_{n}, k_{n}\right)$ with state $( \pm 2)$, modifying their state to \pm 1 , inserting them into $L_{ \pm 1}$ and assigning them values $\phi_{i_{n}, j_{n}, k_{n}}^{n+1}=\phi_{i, j, k}^{n+1} \pm \Delta l$. By repeating this procedure for many time steps the front is propagated any desired total time $t_{\max }$ required by the user. In addition to better computational efficiency (due to processing the strictly required points only), the SFM updates $\phi$ precisely correctly for every point during the whole length of a simulation, thus resulting in better accuracy than traditional LS implementations [49]. The previous SFM algorithm has been implemented in a parallel, GPU environment, which further reduces the time of the simulations.

By simulating several basic structures that resemble the corresponding etching experiments, Radjenovic et al. demonstrated the possibility of evolving anisotropic 


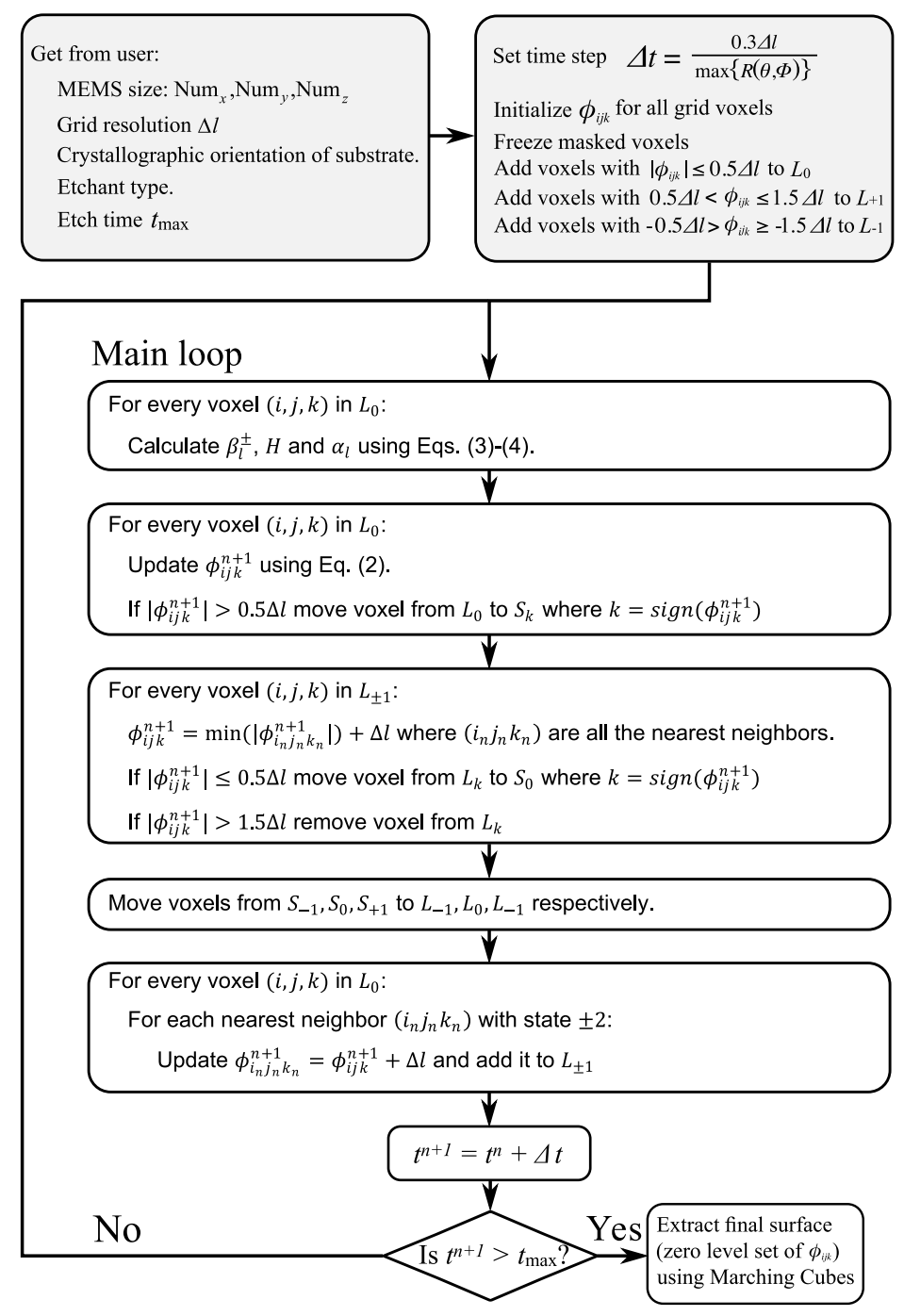

Figure 2. Flow diagram for the LS method implemented in this study (SFM).

etching fronts with the LS method by using 13 experimental etch rates [43, 44, 45, 46]. In contrast, in this study we use a full distribution $R(\theta, \Phi)$ with 8100 experimental etch rates for each etchant [6]. With this experimental input, we are able to explore realistically a wide variety of test cases, comparing the results directly to those from experiments as well as from benchmark simulations, such as the well-established CCA. In addition, we are able to evaluate the simulation of double-side etching, e.g. to produce suspended structures. The coalescing of both top and bottom surfaces is handled inherently by the LS formulation and, consequently, by the SFM implementation. Similarly, our LS simulator enables performing multiple consecutive etchings with the possibility of changing the top and/or bottom masks as well as the etchant. These features, and the required low computational times, make our simulator a versatile tool capable of describing quantitatively many micromachining MEMS processes. 


\section{Results}

This section presents an extensive collection of experimental and simulated results in order to compare the relative merits of the proposed LS implementation, as described in Fig. 2, and a well-established simulator of anisotropic etching, namely, the CCA method. For fairness, we consider the Compensated-Constant Time Stepping (CTCTS) implementation of the CCA method, as described e.g. in Ref. [23], since the LS implementation itself uses a constant time step approach, as described on the left-handside of Fig. 2. Both methods are implemented in CUDA $\mathrm{C}$ and run on a Nvidia GTX 260 device.

\subsection{Orientation dependence of the etch rate}

We first consider the etching of a sphere, which exposes all the crystallographic orientations of the substrate to the etchant. Fig. 3 compares the experimental and simulated stereographic projections of the etch rate for silicon and quartz hemispheres etched in five different etching conditions, including (a) silicon in $\mathrm{KOH} 24 \mathrm{wt} \%$ at $70^{\circ} \mathrm{C}$, (b) silicon in $\mathrm{KOH} 24 \mathrm{wt} \%+\mathrm{IPA}\left(1 \mathrm{~cm}\right.$ satur.) at $65^{\circ} \mathrm{C}$, (c) silicon in TMAH $20 \mathrm{wt} \%$ at $60^{\circ} \mathrm{C}$, (d) silicon in an isotropic etchant and (e) quartz in saturated $\mathrm{NH}_{4} \mathrm{HF}_{2}$ at $70^{\circ} \mathrm{C}$. The simulated distributions have been obtained with a surface grid of $300 \times 300$ voxels and $181 \times 256$ Unit Cells (UCs) for the LS and CCA methods, respectively. For the LS simulations the $\alpha_{F}$ value has been selected as follows: 0.48 for experiments a) and $\mathrm{b}$ ), 0.45 for c) and 0.5 for e). In addition, all simulations have been performed using $\Delta N_{l}=0.01$. In comparison to the experimental distributions, the LS simulations describe accurately the etch rate anisotropy for all five cases, confirming the correctness of our implementation and validating the simulation of dramatically different etchants with the LS method. In comparison, the CCA results seem slightly more noisy in all the cases, but specially for the isotropic etchant and the etching solution applied to quartz. The crystallographic structure of quartz is more complicated than that of silicon, leading

to a more complex atom removal sequence [34]. This results in a more complex surface morphology for the quartz sphere and, thus, the etch rate distribution becomes noisier when sampling the advancement of the etch front over various locations on the sphere.

\subsection{Resolution}

The underlying grid in the LS simulations effectively models the etched substrate and its resolution affects the final accuracy of the results. Typically, the larger the number of grid points the better the accuracy, a phenomenon that is also observed in atomistic models. To quantify this effect, we consider a rather complex dual-axis microprobe structure [50]. As shown in Fig. 4, measurements of the width of the narrowest beam developed in the simulated structure indicate a significant dependence on the grid resolution. In particular, the beam width decreases exponentially as the number of grid points increases (voxels in LS and atoms in CCA), effectively saturating at 11.0 


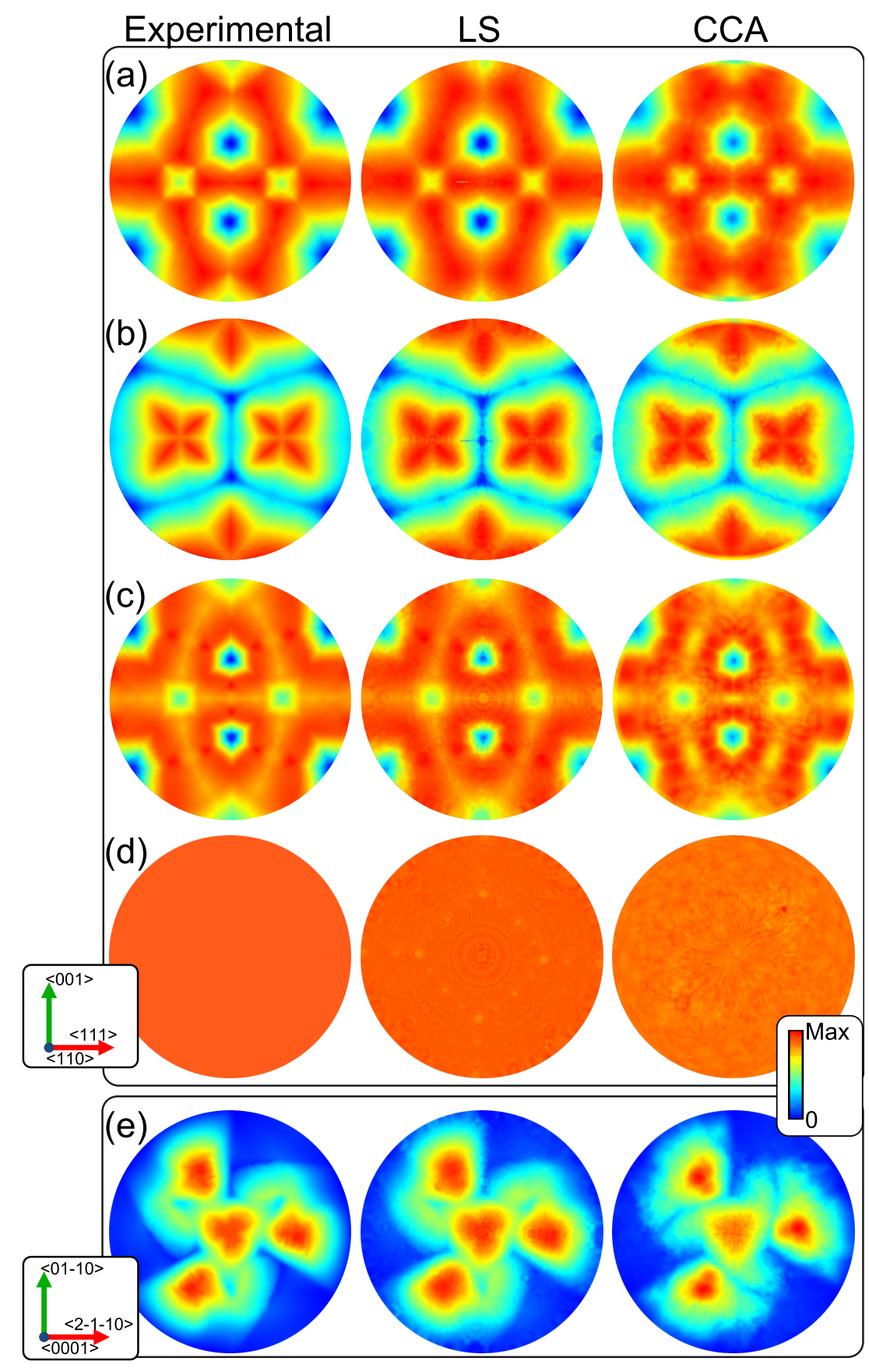

Figure 3. Comparison between experimental and simulated etch rate distributions using the LS and CCA methods: (a) $\mathrm{KOH} 24 \mathrm{wt} \%$ at $70^{\circ} \mathrm{C}$, (b) $\mathrm{KOH} 24 \mathrm{wt} \%+\mathrm{IPA}$ $\left(1 \mathrm{~cm}\right.$ satur.) at $65^{\circ} \mathrm{C}$, (c) TMAH $20 \mathrm{wt} \%$ at $60^{\circ} \mathrm{C}$, (d) an isotropic etchant and (e) saturated $\mathrm{NH}_{4} \mathrm{HF}_{2}$ at $70^{\circ} \mathrm{C}$. (a)-(d) Silicon substrate. (e) Quartz substrate. 


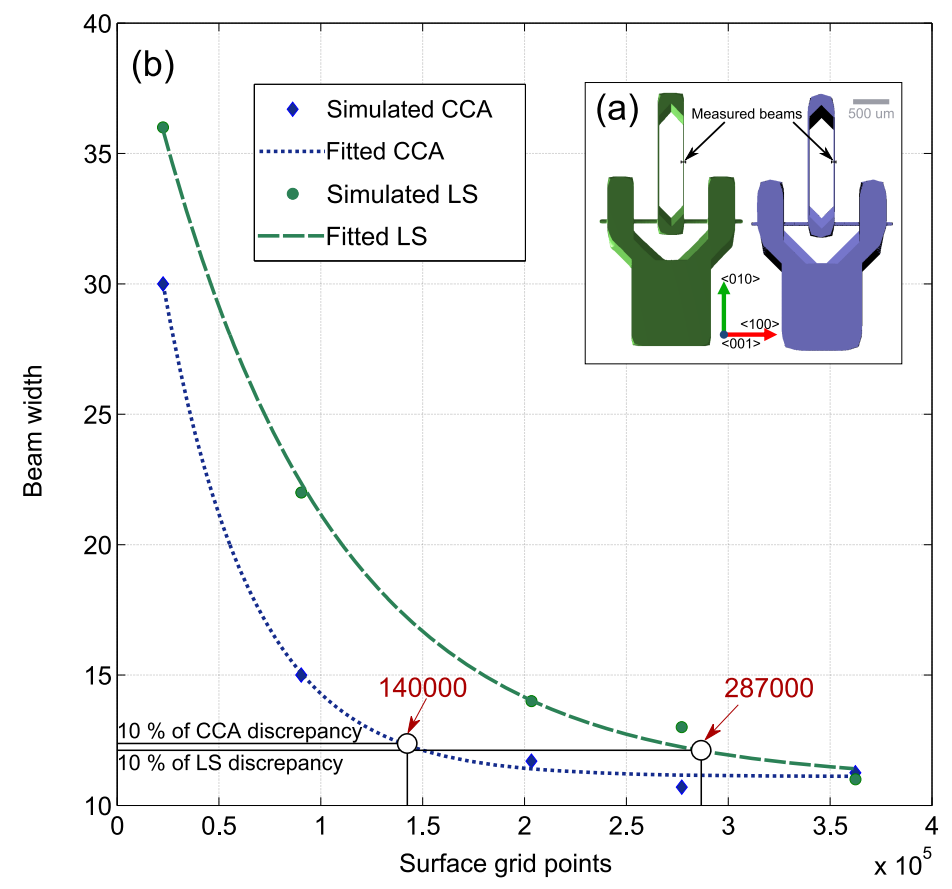

Figure 4. (a) Simulated dual-axis microprobe [50] by the proposed LS implementation (left) and the benchmark CCA method (right). (b) Dependence of the narrowest beam width on resolution. The minimum grid sizes required to obtain a maximum discrepancy of $10 \%$ with respect to the width at the $800 \times 453$ grid are shown.

and $11.25 \mu \mathrm{m}$ for LS and CCA, respectively, for the $800 x 453$ surface grid size. Since the discrepancy between the converged beam widths is only $0.25 \mu \mathrm{m}$ in comparison to the large size of the substrate $\left(4000 \times 2266 \mu \mathrm{m}^{2}\right)$, we conclude that the LS method appears to be a suitable alternative for the simulation of anisotropic etching of advanced structures. In contrast to high resolutions, where the differences between both methods are minimal, at low resolutions the discrepancy is higher. Although LS can reach subvoxel precision by definition, the mask transfer does not. Thus, the difference by a single masked voxel can be significant, becoming a relevant limit when choosing the surface grid size. Considering that a $10 \%$ discrepancy with respect to the converged beam width implies a difference of only 1.1 and $1.13 \mu \mathrm{m}$ for LS and CCA, respectively, the fitted exponentials indicate that the minimal grid resolution to achieve this $10 \%$ deviation is about 287000 voxels and $140000 \mathrm{UC}$ (in silicon, each UC is formed by 8 atoms) for LS and CCA, respectively. Thus, for this structure, the LS method requires about double the number of points than the CCA implementation to avoid errors due to grid resolution. Since this is the most complex three-dimensional structure considered in our set of simulations, we conclude that this same grid resolution can be safely used to compare meaningfully the two simulation procedures for all remaining structures. 


\subsection{Comparison to experimental structures}

In this section the LS and CCA methods are compared in realistic scenarios. Direct comparison with experiments is shown, including measurements of certain structural features. For this purpose we consider several MEMS structures obtained by using silicon and quartz substrates and different etchants, as summarized in Fig. 5. From left to right the columns correspond to (i) the required mask patterns, (ii) the experimental results, (iii) the LS-simulated fronts and (iv) the CCA-based results. For completeness, Table 1 summarizes the details of the simulations, including the crystallographic orientation of the substrate, etchant, substrate size, etching time (for every etching step, if more than one step), the grid size (resolution) used in both simulation models and the computational time required by both methods.

According to Fig. 5, the structures obtained by LS and CCA are very similar. This is confirmed by the measurements shown on the corresponding pictures, where the differences between both simulators are of the order of a few to several tens of microns while the substrates measure several hundred to even a few thousand microns. The feature dimensions shown in red on the experimental images have been obtained by using the scale attached to each figure. The maximum discrepancies occur at the smallest parts, such as the tips in experiments (a) and (b), where the two models differ by as much as $6.87 \mu \mathrm{m}$ and $17.4 \mu \mathrm{m}$, respectively. However, these errors can be minimized by simulating exclusively those particular parts of the structures with higher resolution. In contrast to complex MEMS structures, such as experiments (a), (b), (c), (g) and (h), a much smaller grid can be used without sacrificing the accuracy in the case of simpler topologies with no sensitive details, such as experiments (d), (e) and (f). Nevertheless, we have used a larger grid size for these systems in order to be consistent with the rest of the tests. The following is a more detailed description of the similarities and discrepancies between the experiments and simulations:

- Fig. 5 (a): Although no information about the etching steps or feature dimensions is provided by the source [58], the results demonstrate that a similar structure can be achieved by both simulators by appropriately selecting the parameters, i.e. the etchant, concentration, temperature, mask patterns and process times.

- Fig. 5 (b): The two simulated shapes are very similar to the experimental structure. Due to the perspective of the experimental image it is difficult to determine the beam width. However, its length is about $2000 \mu m$, in good agreement with both simulated values. The tip height obtained by the CCA simulator $(7.2 \mu \mathrm{m})$ resembles better the experiment $(9.1 \mu \mathrm{m})$ than the LS method $(24.6 \mu \mathrm{m})$. This can be minimized by simulating independently the tip region with larger resolution.

- Fig. 5 (c): The experimental scale bar indicates that the width of the inertial mass obtained by CCA $(3086 \mu \mathrm{m})$ is closer to the experiment. Nevertheless, the LS value $(3149 \mu \mathrm{m})$ differs by only $89.3 \mu \mathrm{m}$ while the length of the substrate is $\sim 22000 \mu \mathrm{m}$.

- Fig. 5 (d): Both simulated profiles reproduce well the experimental structure. The error for the shown dimension is only 1.2 and $2.7 \mu \mathrm{m}$ for LS and CCA, respectively. 

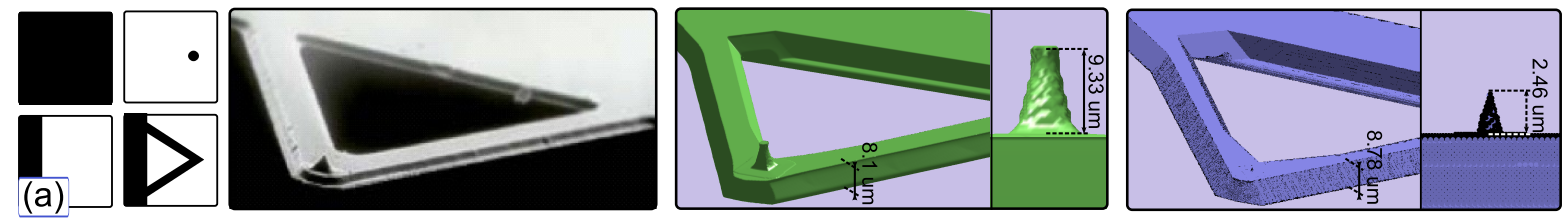

(b)
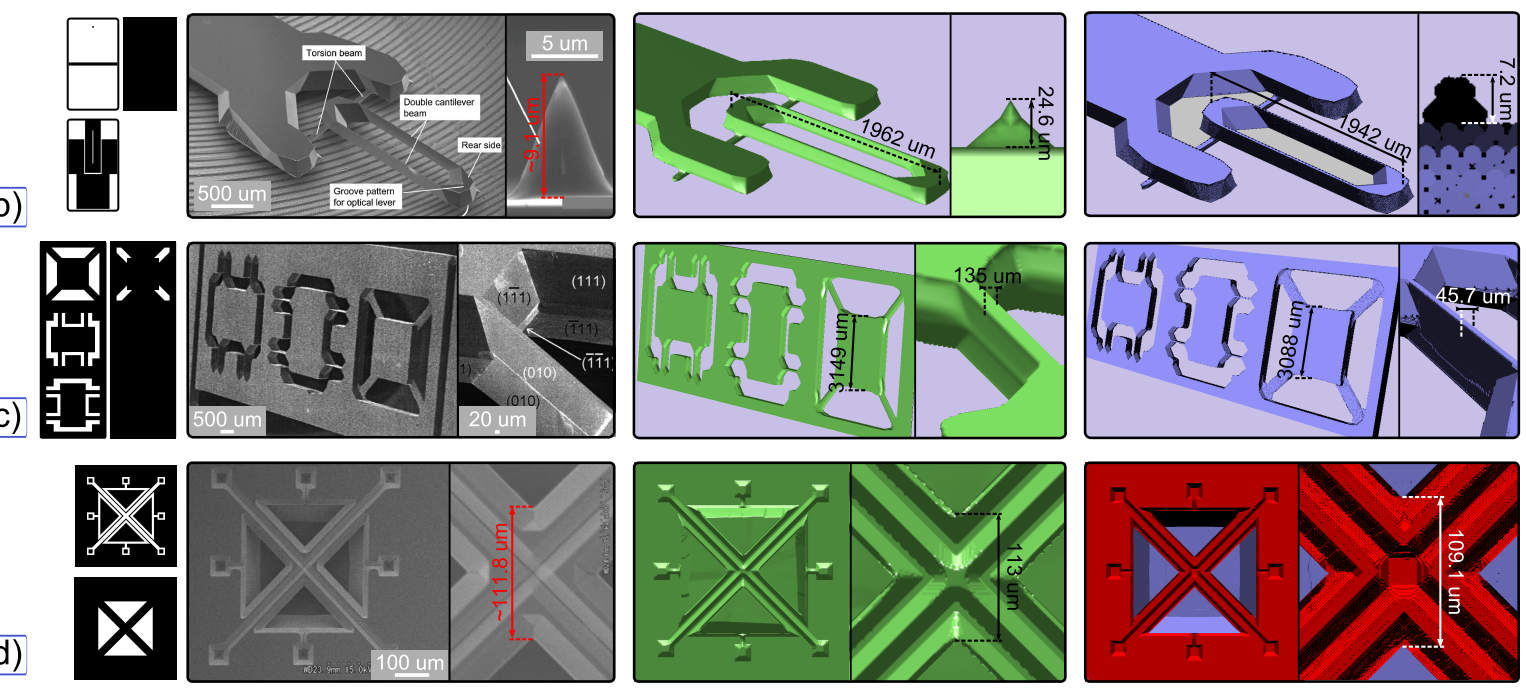

(d)
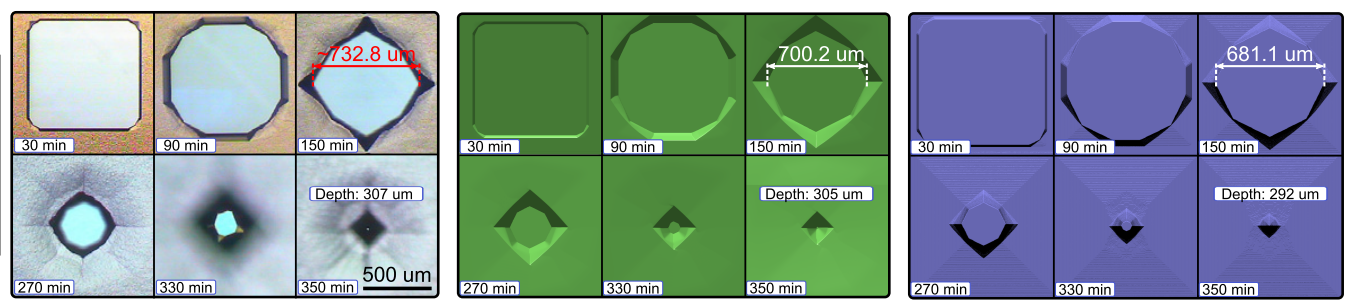

(e)
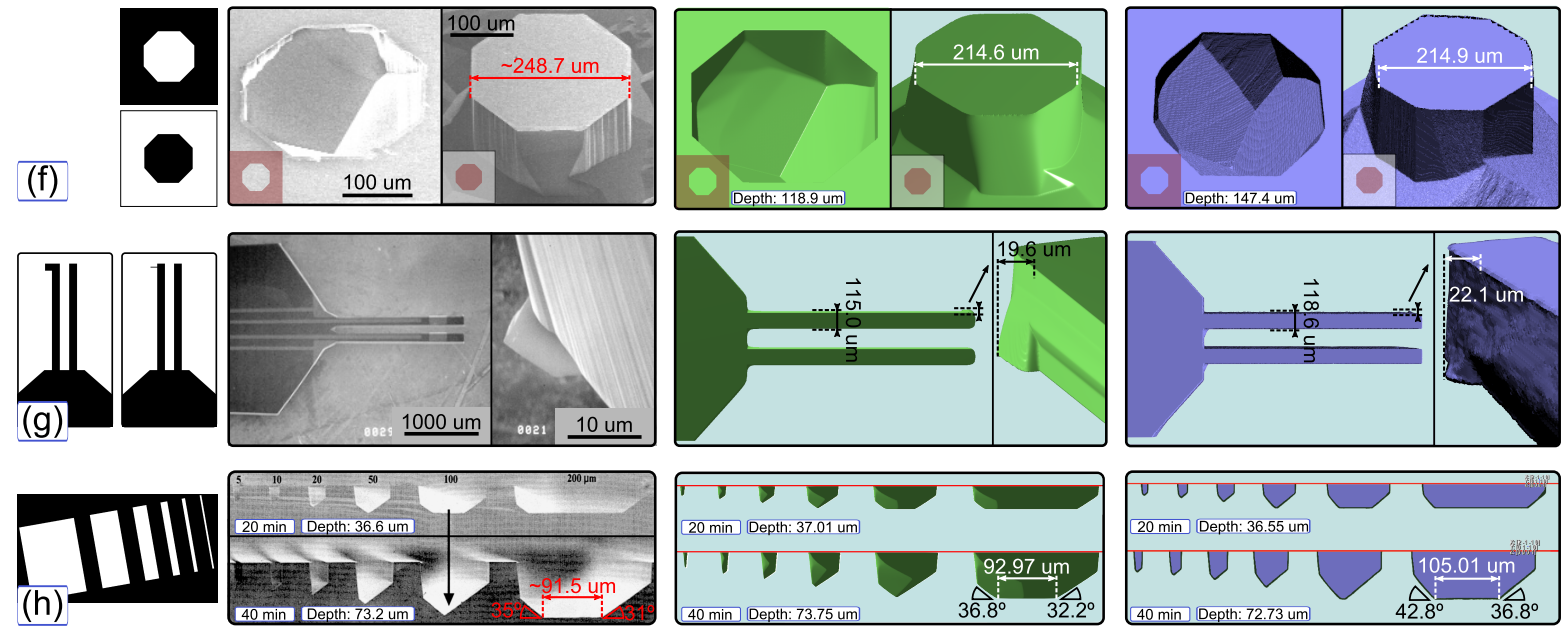

Figure 5. Comparison between simulation and experiment (by columns, left to right): (i) required masks, (ii) experimental images, (iii) LS simulations and (iv) CCA simulations. Experiments on silicon: (a) AFM tip [51], (b) Dual-axis micromechanical probe [52], (c) 3-axis accelerometer [53], (d) suspended microchannel [12] and (e) microneedles [54]. Experiments on quartz: (f) Cavity and mesa [55], (g) Tuningfork probe [56] and (h) grooves [57]. Several length measurements are shown on the experimental and simulated scenes. 
- Fig. 5 (e): This experiment is well reproduced by both simulators. The displayed dimensions demonstrate that the LS result is closer to the experiment, specially the depth, which only differs by $2 \mu \mathrm{m}$ in comparison to the large substrate size $\left(1000 \times 1000 \mu m^{2}\right)$.

- Fig. 5 (f): The simulated shapes are very similar to the experiments. Although both simulators generate a similar width for the mesa structure, they differ by about $34 \mu \mathrm{m}$ from the experimental mesa.

- Fig. 5 (g): The simulated structures are very similar to each other and also to the experiment. Due to the perspective of the experimental closeup (right-hand-side image) it is difficult to obtain the tip length $(<10 \mu \mathrm{m})$. Although the LS value is smaller than that for CCA (19.6 vs $22.1 \mu \mathrm{m}$ ) and, thus, closer to the experiment, the overall CCA shape is more similar to the experiment.

- Fig. 5 (h): The depths reached by both simulators are in close proximity to the experiment. In this particular case, the largest cavity measurements show that the LS method provides more accurate results.

In terms of computational performance, our parallel SFM implementation is 1.1-10 times faster than the CCA method. However, the CCA method is 1.2 times faster than the LS implementation for the left-hand-side experiment in (f). Although a traditional sequential implementation of both methods shows that the CCA is twice as fast [59], in this study the GPU implementation of the CCA makes use of an octree data structure, which reduces the memory use but adds a relevant overhead to the GPU calculations [24]. The computational cost of our LS implementation is primarily due to the calculation of the spatial derivatives, the local etch rate and the maximum in Eq. 4, which altogether represent $56 \%$ of the total computational time on average. In addition, steps (2), (3) and (4) of Fig. 2 are also relevant, representing 15.1\%, 12.6\% and 11.4\% of the computational time, respectively, while the final step (5) only represents $4.9 \%$ of the global time.

Table 1. Experimental conditions and simulation parameters used in Fig. 5.

\begin{tabular}{|c|c|c|c|c|c|c|c|c|}
\hline \multirow{2}{*}{ Experiment } & \multicolumn{4}{|c|}{ Experiment features } & \multicolumn{2}{|c|}{ Surface grid size } & \multicolumn{2}{|c|}{$\begin{array}{l}\text { Simulation } \\
\text { time (s) }\end{array}$} \\
\hline & $\begin{array}{l}\text { Substrate } \\
\text { and orientation }\end{array}$ & Etchant & $\begin{array}{l}\text { Substrate } \\
\text { size }(\mu m)\end{array}$ & $\begin{array}{c}\text { Etching } \\
\text { time }(\min )\end{array}$ & $\begin{array}{c}\text { LS } \\
\text { (voxels) }\end{array}$ & $\begin{array}{c}\text { CCA } \\
\text { (atoms) }\end{array}$ & LS & CCA \\
\hline Fig. 5 (a) & Silicon $(100),\langle 100\rangle$ flat & $\mathrm{KOH} 40 \mathrm{wt} \% 70^{\circ} \mathrm{C}$ & $250 \times 250 \times 22$ & $17+11+7$ & $536 \times 536$ & $375 \times 375$ & 8.1 & 70.2 \\
\hline Fig. 5 (b) & Silicon $(100),\langle 100\rangle$ flat & $\mathrm{KOH} 40 \mathrm{wt} \% 70^{\circ} \mathrm{C}$ & $2266 \times 4000 \times 190$ & $80+270$ & $536 \times 536$ & $375 \times 375$ & 8.0 & 74.5 \\
\hline Fig. 5 (c) & Silicon $(100),\langle 100\rangle$ flat & $\mathrm{KOH} 40 \mathrm{wt} \% 70^{\circ} \mathrm{C}$ & $21546 \times 7182 \times 399$ & $20+45$ & $928 \times 309$ & $648 \times 216$ & 2.1 & 21.7 \\
\hline Fig. 5 (d) & Silicon $(100),\langle 110\rangle$ flat & $\begin{array}{l}\text { TMAH } 25 \mathrm{wt} \% 80^{\circ} \mathrm{C} / \\
\text { Triton } 0.1 \mathrm{v} / \mathrm{v} \text { addition }\end{array}$ & $800 \times 800$ & $40+160$ & $536 \times 536$ & $375 \times 375$ & 32.8 & 36.9 \\
\hline Fig. 5 (e) & Silicon $(100),\langle 110\rangle$ flat & $\mathrm{KOH} 30 \mathrm{wt} \% 80^{\circ} \mathrm{C}$ & $1000 \times 1000$ & From 30 to 350 & $536 \times 536$ & $375 \times 375$ & 63.2 & 157.9 \\
\hline Fig. 5 (f) & Quartz (0001) & $\mathrm{NH}_{4} \mathrm{HF}_{2} 85^{\circ} \mathrm{C}$ & $444 \times 444$ & 81.5 & $536 \times 536$ & $403 \times 348$ & $44.5 / 52.2$ & $37.1 / 125.5$ \\
\hline Fig. 5 (g) & Quartz (0001) & $\mathrm{NH}_{4} \mathrm{HF}_{2} 85^{\circ} \mathrm{C}$ & $1353 \times 2459 \times 100$ & 100 & $398 \times 723$ & $259 \times 545$ & 9.7 & 18.6 \\
\hline Fig. 5 (h) & Quartz (0001) & $\mathrm{NH}_{4} \mathrm{HF}_{2} 85^{\circ} \mathrm{C}$ & $723 \times 390$ & 20,40 & $729 \times 393$ & $475 \times 295$ & 16.8 & 28.0 \\
\hline
\end{tabular}

Overall, Fig. 5 demonstrates the reliability of the proposed LS simulator, which is capable of achieving similar accuracy while producing less noisy results than the CCA 
model. Although the CCA method provides better accuracy at small parts, the LS implementation achieves better results for several systems, such as (e) and (h). Thus, we conclude that the proposed parallel SFM implementation of the LS method has a large potential to become a tool for design of complex MEMS structures.

\section{Conclusions}

Based on the Level Set (LS) method, a fully-operational simulator of anisotropic wet chemical etching is developed for a parallel, Graphics Processing Unit (GPU) environment. The aim is to provide a versatile tool for the design of Micro-ElectroMechanical-Systems (MEMS) based on the inherent capability of the LS method to simulate (i) the splitting and coalescing of disjoint regions of the front, such as in double-side etching, and (ii) new etchants and/or substrate materials without any need for recalibrating the internal parameters of the method. Based on the increased computational efficiency and accuracy with respect to the conventional LS method, an implementation based on the Sparse Field Method (SFM) is presented, comparing its performance to that from the state-of-the-art, atomistic method -the Continuous Cellular Automaton (CCA)- for a wide variety of experimental conditions, including silicon and quartz substrates in different etchants, such as $\mathrm{KOH}, \mathrm{KOH}+\mathrm{IPA}$, TMAH and TMAH+triton for silicon and $\mathrm{NH}_{4} \mathrm{HF}_{2}$ for quartz.

Although for highly anisotropic etchants LS tends to soften the corners and edges, reducing slightly the accuracy, and the method typically requires slightly larger grids than CCA, the differences between the simulated features by LS and CCA are of the order of several microns for substrates measuring even a few millimeters, concluding that the LS implementation achieves similar accuracy as the CCA method with less fluctuations in the etch front. Due to the strong, parallel nature of the LS method and the high computational efficiency of the currently available, many-core platforms, such as Nvidia's GPUs, our parallel SFM implementation is typically faster than the CCA method. This feature is assigned to the use of an octree data structure in the CCA method, which reduces memory allocation but requires additional calculations and management. In addition to the higher computational performance, the largest strengths of our SFM implementation are: (i) the absence of a time-consuming calibration procedure prior to performing the simulations, which is strictly necessary in the CCA approach when the etchant is modified, and (ii) the direct application of the simulation tool to any type of substrate, which typically requires a dedicated effort to analyze and classify the different atomistic neighborhoods in the CCA approach.

\section{Acknowledgements}

This work has been supported by the Spanish FPI-MICINN BES-2011-045940 grant and the Ramón y Cajal Fellowship Program by the Spanish Ministry of Science and Innovation. Also, we acknowledge support by the JAE-Doc grant form the Junta para 
la Ampliación de Estudios program co-funded by FSE and the Professor Partnership Program by NVIDIA Corporation.

\section{References}

[1] Weirauch D F. Correlation of the anisotropic etching of single-crystal silicon spheres and wafers. Journal of Applied Physics, 46(4):1478-1483, 1975.

[2] Seidel H, Csepregi L, Heuberger A, and Baumgartel H. Anisotropic etching of crystalline silicon in alkaline solutions: I . orientation dependence and behavior of passivation layers. Journal of The Electrochemical Society, 137(11):3612-3626, 1990.

[3] Zielke D and Frühauf J. Determination of rates for orientation-dependent etching. Sensors and Actuators A: Physical, 48(2):151-156, 1995.

[4] Wind R A and Hines M A. Macroscopic etch anisotropies and microscopic reaction mechanisms: a micromachined structure for the rapid assay of etchant anisotropy. Surface Science, 460(13):21$38,2000$.

[5] Gosálvez M A, Sato K, Foster A S, Nieminen R M, and Tanaka H. An atomistic introduction to anisotropic etching. Journal of Micromechanics and Microengineering, 17(4):S1-S26, 2007.

[6] Gosálvez M A, Pal P, Ferrando N, Hida H, and Sato K. Experimental procurement of the complete $3 \mathrm{~d}$ etch rate distribution of si in anisotropic etchants based on vertically micromachined wagon wheel samples. Journal of Micromechanics and Microengineering, 21(12):125007, 2011.

[7] Sato K, Shikida M, Matsushima Y, Yamashiro T, Asaumi K, Iriye Y, and Yamamoto M. Characterization of orientation-dependent etching properties of single-crystal silicon: effects of koh concentration. Sensors and Actuators A: Physical, 64(1):87-93, 1998.

[8] Zubel I and Kramkowska M. The effect of alcohol additives on etching characteristics in koh solutions. Sensors and Actuators A: Physical, 101(3):255-261, 2002.

[9] Charbonnieras A R and Tellier C R. Characterization of the anisotropic chemical attack of $\{\mathrm{hk0}\}$ silicon plates in a t.m.a.h. solution: Determination of a database. Sensors and Actuators A: Physical, 77(2):81-97, 1999.

[10] Shikida M, Sato K, Tokoro K, and Uchikawa D. Differences in anisotropic etching properties of koh and tmah solutions. Sensors and Actuators A: Physical, 80(2):179-188, 2000.

[11] Gosálvez M A, Zubel I, and Viinikka E. Wet etching of silicon, Chap. Ed. H. Seidel in Silicon Based MEMS Materials \&3 Technologies, Part 4, Chapter 24, Book Ed. V. Lindroos, M. Tilli A. Lehto and T. Motooka. Micro \& Nano Technologies. William Andrew/Elsevier, 2010.

[12] Pal P and Sato K. Various shapes of silicon freestanding microfluidic channels and microstructures in one-step lithography. http://dx.doi.org/10.1088/0960-1317/19/5/055003. Journal of Micromechanics and Microengineering, 19(5):055003, 2009.

[13] Pal P, Gosálvez M A, and Sato K. Silicon micromachining based on surfactant-added tetramethyl ammonium hydroxide: Etching mechanism and advanced applications. Japanese Journal of Applied Physics, 49(5):056702, 2010.

[14] Zubel I and Kramkowska M. Etch rates and morphology of silicon (h k l) surfaces etched in koh and koh saturated with isopropanol solutions. Sensors and Actuators A: Physical, 115(23):549-556, 2004.

[15] Fruhauf J, Trautmann K, Wittig J, and Zielke D. A simulation tool for orientation dependent etching. Journal of Micromechanics and Microengineering, 3(3):113-115, 1993.

[16] Asaumi K, Iriye Y, and Sato K. Anisotropic-etching process simulation system microcad analyzing complete $3 \mathrm{~d}$ etching profiles of single crystal silicon. In Workshop on Micro Electro Mechanical Systems. MEMS '97, Proceedings, IEEE., Tenth Annual International, pages 412-417, jan 1997.

[17] Than O and Büttgenbach S. Simulation of anisotropic chemical etching of crystalline silicon using a cellular automata model. Sensors and Actuators A: Physical, 45(1):85-89, 1994.

[18] Camon H, Gue A M, Daniel J S, and Djafari-Rouhani M. Modelling of anisotropic etching in silicon-based sensor application. Sensors and Actuators A, 33(1):103-105, 1992. 
[19] Hubbard T and Antonsson E K. Cellular automata modeling in mems design. Sensors and Materials, 9:, 1997.

[20] Gosálvez M A, Nieminen R M, Kilpinen P, Haimi E, and Lindroos V. Anisotropic wet chemical etching of crystalline silicon: atomistic monte-carlo simulations and experiments. Applied Surface Science, 178(14):7-26, 2001.

[21] Zhu Z and Liu C. Micromachining process simulation using a continuous cellular automata method. Journal of Microelectromechanical Systems, 9(2):252-261, june 2000.

[22] Gosálvez M A, Xing Y, and Sato K. Analytical solution of the continuous cellular automaton for anisotropic etching. Journal of Micromechanical Systems, 17(2):410-431, april 2008.

[23] Ferrando N, Gosálvez M A, Cerdá J, Gadea R, and Sato K. Faster and exact implementation of the continuous cellular automaton for anisotropic etching simulations. Journal of Micromechanics and Microengineering, 21(2):025021, 2011.

[24] Ferrando N, Gosálvez M A, Cerdá J, Gadea R, and Sato K. Octree-based, gpu implementation of a continuous cellular automaton for the simulation of complex, evolving surfaces. Computer Physics Communications, 182(3):628-640, 2011.

[25] Moktadir Z and Camon H. Monte carlo simulation of anisotropic etching of silicon: investigation of $\langle 111\rangle$ surface properties. Modelling and Simulation in Materials Science and Engineering, 5(5):481-488, 1997.

[26] Flidr J, Huang Y-C, and Melissa A H. An atomistic mechanism for the production of twoand three-dimensional etch hillocks on si(111) surfaces. The Journal of Chemical Physics, 111(15):6970-6981, 1999.

[27] Gosálvez M A and Nieminen R M. Surface morphology during anisotropic wet chemical etching of crystalline silicon. New Journal of Physics, 5(1):100.1-100.28, 2003.

[28] Xing Y, Gosálvez M A, Sato K, Tian M, and Yi H. Evolutionary determination of kinetic monte carlo rates for the simulation of evolving surfaces in anisotropic etching of silicon. Journal of Micromechanics and Microengineering, 22(8):085020, 2012.

[29] Xing Y, Gosálvez M A, Sato K, and Yi H. Orientation-dependent surface morphology of crystalline silicon during anisotropic etching using a continuous cellular automaton. Journal of Micromechanics and Microengineering, 20(1):015019, 2010.

[30] Zhou Z F, Huang Q A, Li W H, and Deng W. A cellular automaton-based simulator for silicon anisotropic etching processes considering high index planes. Journal of Micromechanics and Microengineering, 17(4):S38, 2007.

[31] IntelliSense-Corp. Intellietch webpage. http://www.intellisense.com/modules/IntelliEtch . html, aug 2011.

[32] Gosálvez M A, Xing Y, Sato K, and Nieminen R M. Discrete and continuous cellular automata for the simulation of propagating surfaces. Sensors and Actuators A: Physical, 155(1):98-112, 2009.

[33] Gosálvez M A, Ferrando N, Xing Y, Pal P, and Sato K. Simulating anisotropic etching of silicon in any etchant: evolutionary algorithm for the calibration of the continuous cellular automaton. Journal of Micromechanics and Microengineering, 21:065017, 2011.

[34] Ferrando N, Gosálvez M A, and Colom R J. Evolutionary continuous cellular automaton for the simulation of wet etching of quartz. Journal of Micromechanics and Microengineering, 22:025021, 2012.

[35] Osher S and Sethian J A. Fronts propagating with curvature-dependent speed: Algorithms based on hamilton-jacobi formulations. Journal of Computational Physics, 79(1):12-49, 1988.

[36] Adalsteinsson D and Sethian J A. A level set approach to a unified model for etching, deposition, and lithography i: Algorithms and two-dimensional simulations. Journal of Computational Physics, 120(1):128-144, 1995.

[37] Adalsteinsson D and Sethian J A. A level set approach to a unified model for etching, deposition, and lithography ii: Three-dimensional simulations. Journal of Computational Physics, 122(2):348-366, 1995. 
[38] Adalsteinsson D and Sethian J A. A level set approach to a unified model for etching, deposition, and lithography iii: Redeposition, reemission, surface diffusion, and complex simulations. Journal of Computational Physics, 138(1):193-223, 1997.

[39] Ertl O and Selberherr S. A fast level set framework for large three-dimensional topography simulations. Computer Physics Communications, 180(8):1242-1250, 2009.

[40] Ertl O and Selberherr S. Three-dimensional level set based bosch process simulations using ray tracing for flux calculation. Microelectronic Engineering, 87(1):20-29, 2010.

[41] Burzynski T and Papini M. Level set methods for the modelling of surface evolution in the abrasive jet micromachining of features used in mems and microfluidic devices. Journal of Micromechanics and Microengineering, 20(8):085004, 2010.

[42] Radjenovic B, Lee J K, and Radmilovic-Radjenovic M. Sparse field level set method for non-convex hamiltonians in $3 \mathrm{~d}$ plasma etching profile simulations. Computer Physics Communications, 174(2):127-132, 2006.

[43] Radjenovic B, Radmilovic-Radjenovic M, and Mitric M. Nonconvex hamiltonians in three dimensional level set simulations of the wet etching of silicon. Applied Physics Letters, 89(21):213102 -213102-2, nov 2006.

[44] Radjenovic B and Radmilovic-Radjenovic M. Level set simulations of the anisotropic wet etching process for device fabrication in nanotechnologies. Hemijska industrija, 64(2):93-97, 2010.

[45] Radjenovic B, Radmilovic-Radjenovic M, and Mitric M. Level set approach to anisotropic wet etching of silicon. Sensors, 10(5):4950-4967, 2010.

[46] Radjenovic B and Radmilovic-Radjenovic M. Three-dimensional simulations of the anisotropic etching profileevolution for producing nanoscale devices. Acta Physica Polonica A, 119(3):447450, 2011.

[47] Crandall M G and Lions P L. Two approximations of solutions of hamilton-jacobi equations. Mathematics of Computation, 43(167):1-19, 1984.

[48] Whitaker R T. A level-set approach to 3d reconstruction from range data. International Journal of Computer Vision, 29:203-231, 1998.

[49] Gomes J and Faugeras O. Reconciling distance functions and level sets. Journal of Visual Communication and Image Representation, 11:209-223, 1999.

[50] Fukuzawa K, Terada S, Shikida M, Amakawa H, Zhang H, and Mitsuya Y. Mechanical design and force calibration of dual-axis micromechanical probe for friction force microscopy. Journal of Applied Physics, 101(3):034308, 2007.

[51] Reproduced with permission of prof. Liu C, from [58].

[52] Reprinted with permission from [50]. Copyright 2013, American Institute of Physics.

[53] Schröpfer G, Labachelerie M de, Ballandras S, and Blind P. Collective wet etching of a 3d monolithic silicon seismic mass system. http://dx.doi.org/10.1088/0960-1317/8/2/008. Journal of Micromechanics and Microengineering, 8(2):77, 1998.

[54] Wilke N, Reed M L, and Morrissey A. The evolution from convex corner undercut towards microneedle formation: theory and experimental verification. http://dx.doi.org/10.1088/ 0960-1317/16/4/018. Journal of Micromechanics and Microengineering, 16(4):808, 2006.

[55] Reproduced with permission of prof. Hung M-T, from [60].

[56] Reprinted from [61], Copyright 2013, with permission from Elsevier.

[57] Liang J, Kohsaka F, Matsuo T, and Ueda T. Wet etched high aspect ratio microstructures on quartz for mems applications. http://dx.doi.org/10.1541/ieejsmas.127.337. (C)IEEJ Transactions on Sensors and Micromachines, 127(7):337-342, 2007.

[58] Liu C. Fundations of MEMS. Illinois Ece Series. Prentice Hall, 2006.

[59] Montoliu C, Ferrando N, Gosálvez M A, Cerdá J, and Colom R J. Implementation and evaluation of the level set method: Towards efficient and accurate simulation of wet etching for microengineering applications. Submitted to Computer Physics Communications, 2013.

[60] Chih hao C and Hung M-T. A study of etch the anisotropic of quartz microstructures. Master's thesis, Department of Mechanical Engineering, National Central University, Taiwan, 2011. 
[61] Hida H, Shikida M, Fukuzawa K, Murakami S, Sato Ke, Asaumi K, Iriye Y, and Sato Ka. Fabrication of a quartz tuning-fork probe with a sharp tip for afm systems. Sensors and Actuators A: Physical, 148(1):311-318, 2008. 